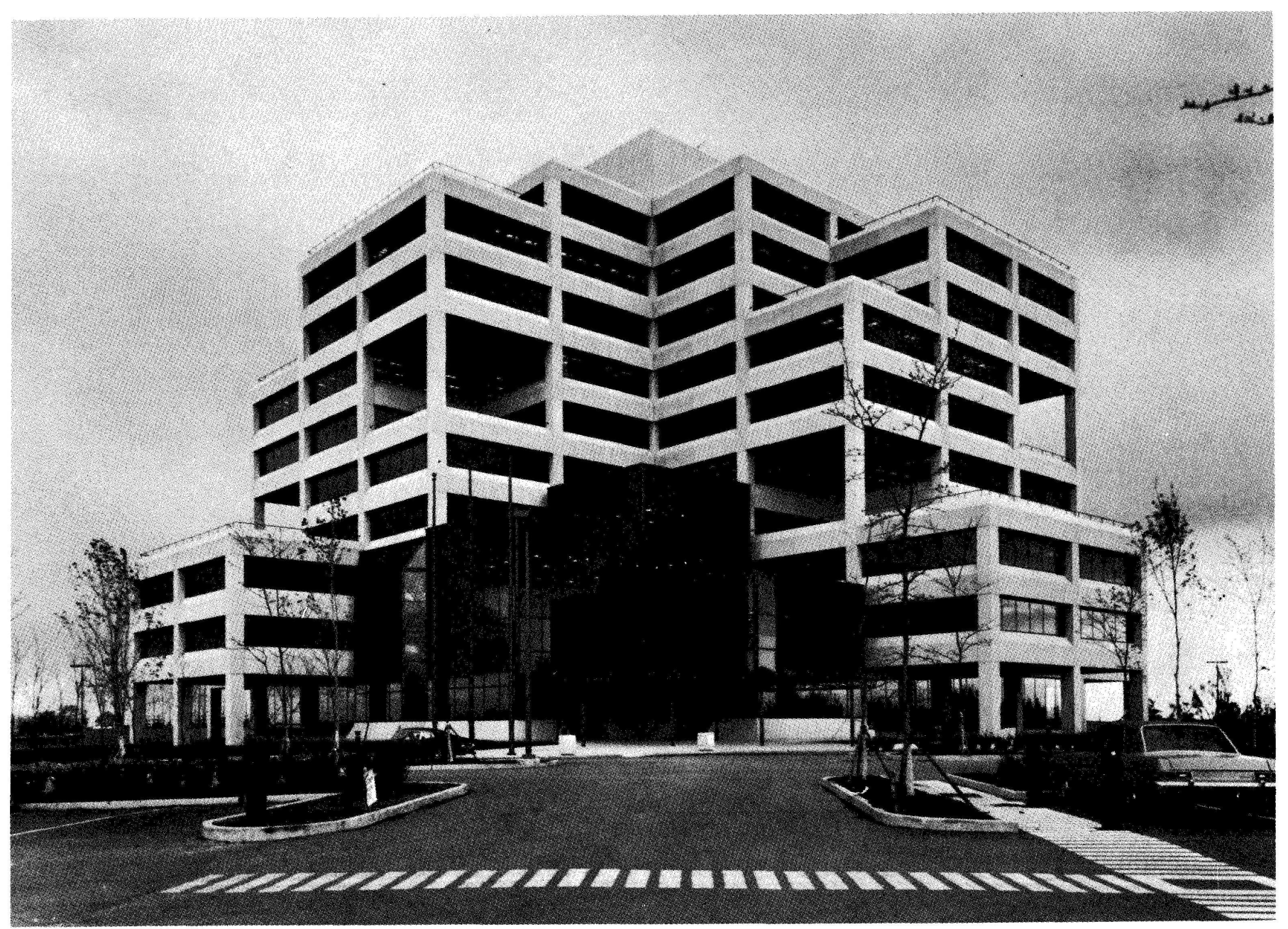

\title{
Centro Federal de Comunidad Saint Louis - EE.UU.
}

Hellmuth, Obata y Kassabaum, arquitectos

145-106

\section{situación}

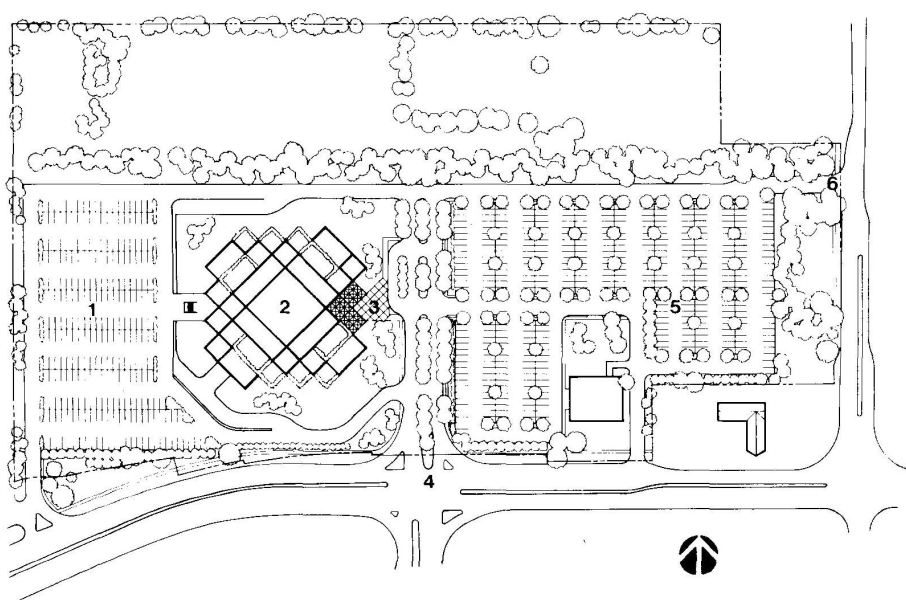

1. Segundo nivel de aparcamiento; 2 . Sede administrativa; 3. Entrada; 4. Entrada al aparcamiento; 5. Aparcamiento; 6. Entrada secundaria al aparcamiento.

\begin{abstract}
sinopsis
El Community Federal Center está emplazado en las afueras de la ciudad de Saint Louis. Fue concebido y diseñado por los autores del proyecto -en un estilo que les es característico-. como un complejo arquitectónico suburbano destinado a oficinas.

La obra, que se eleva sobre un terreno despejado, es amplia de base, presentando un volu-
men que no es ni la torre propia del centro urbano ni la clásica construcción rural de baja altura.

EI lenguaje arquitectónico del edificio permite una integración, por simpatía, en el contexto de la zona de viviendas unifamiliares cercana, sin llegar, naturalmente, a asimilarse a ellas dada la disparidad de funciones. Se lleva a cabo por medio de una fractura de volúmenes. escalonados, que rompen la frialdad clásica del gran edificio de oficinas, sin dejar de indicar, por su robustez, la actividad que alberga en su interior.
\end{abstract}




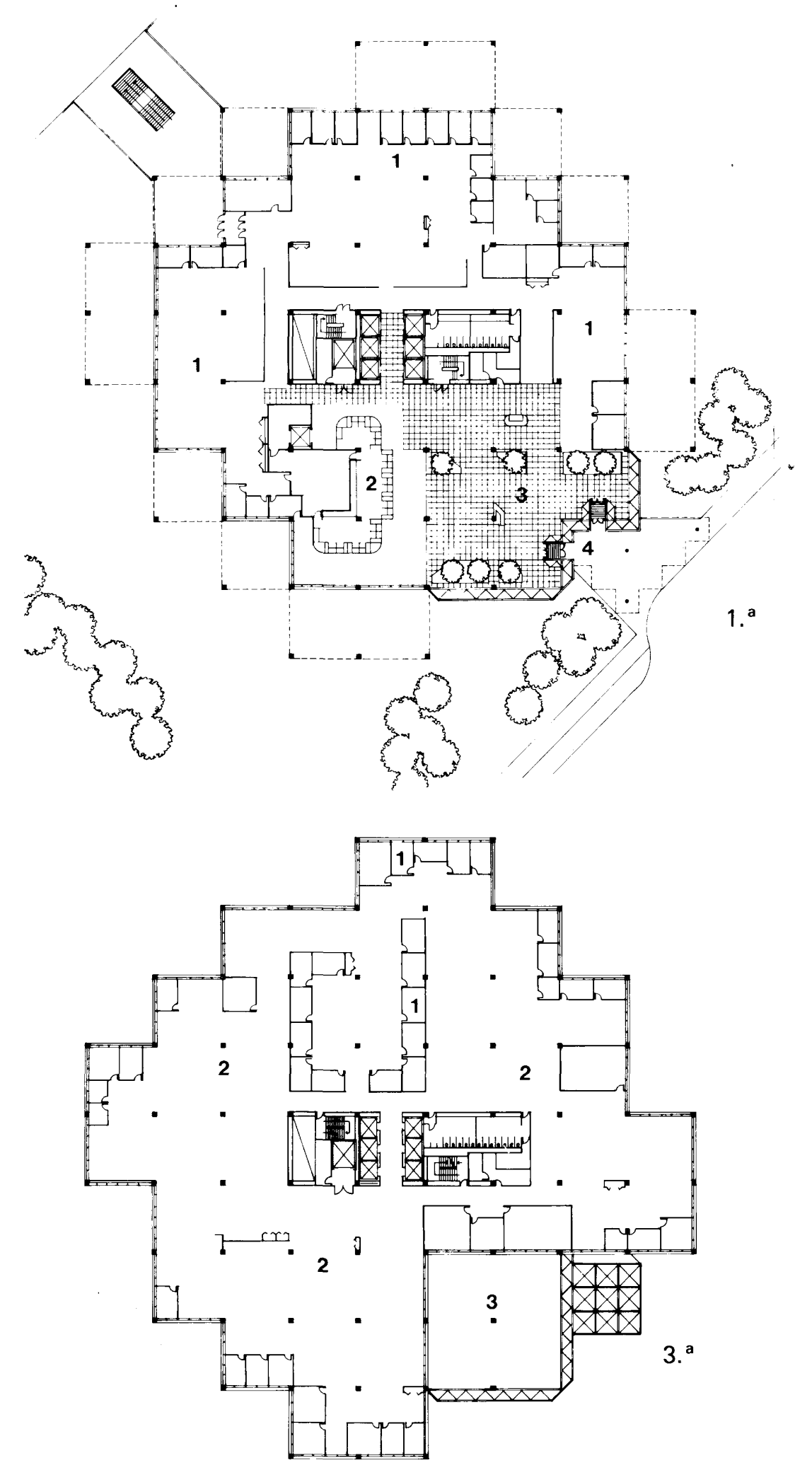

\section{plantas}

\section{primera}

1. Oficinas del Banco; 2. Cajas; 3. Patio ajardinado; 4. Entrada.

tercera

1. Oficinas privadas; 2 . Oficinas abiertas; 3. Atrio.

\section{décima}

1. Terrazas; 2. Despachos; 3. Recepción; 4. Sala de juntas.

La organización de la última de las diez plantas de que consta el edificio, pone de manifiesto la simplicidad a que aludíamos: se trata de despachos dispuestos en cruz, exactamente iguales entre sí, con un cuerpo central de enlaces verticales y servicios. La variedad y el movimiento están dados por medio de las terrazas, que rompen la apariencia simétrica, dando, por el contrario, la imagen opuesta de cubos superpuestos irregularmente. A este mismo fin contribuyen los cambios en el perímetro de fachada de una planta a otra, conformando terrazas que enriquecen notablemente el ámbito aburrido de las oficinas, creando rincones agradables en el interior, y dotando a despachos y zonas de recreo - como la cafetería - de espacios libres.

En conjunto el complejo emplea una edificabilidad de $28.000 \mathrm{~m}^{2}$, de los cuales 18.500 están ocupados por las oficinas centrales del banco, y los 9.500 restantes arrendados a otras sociedades. 

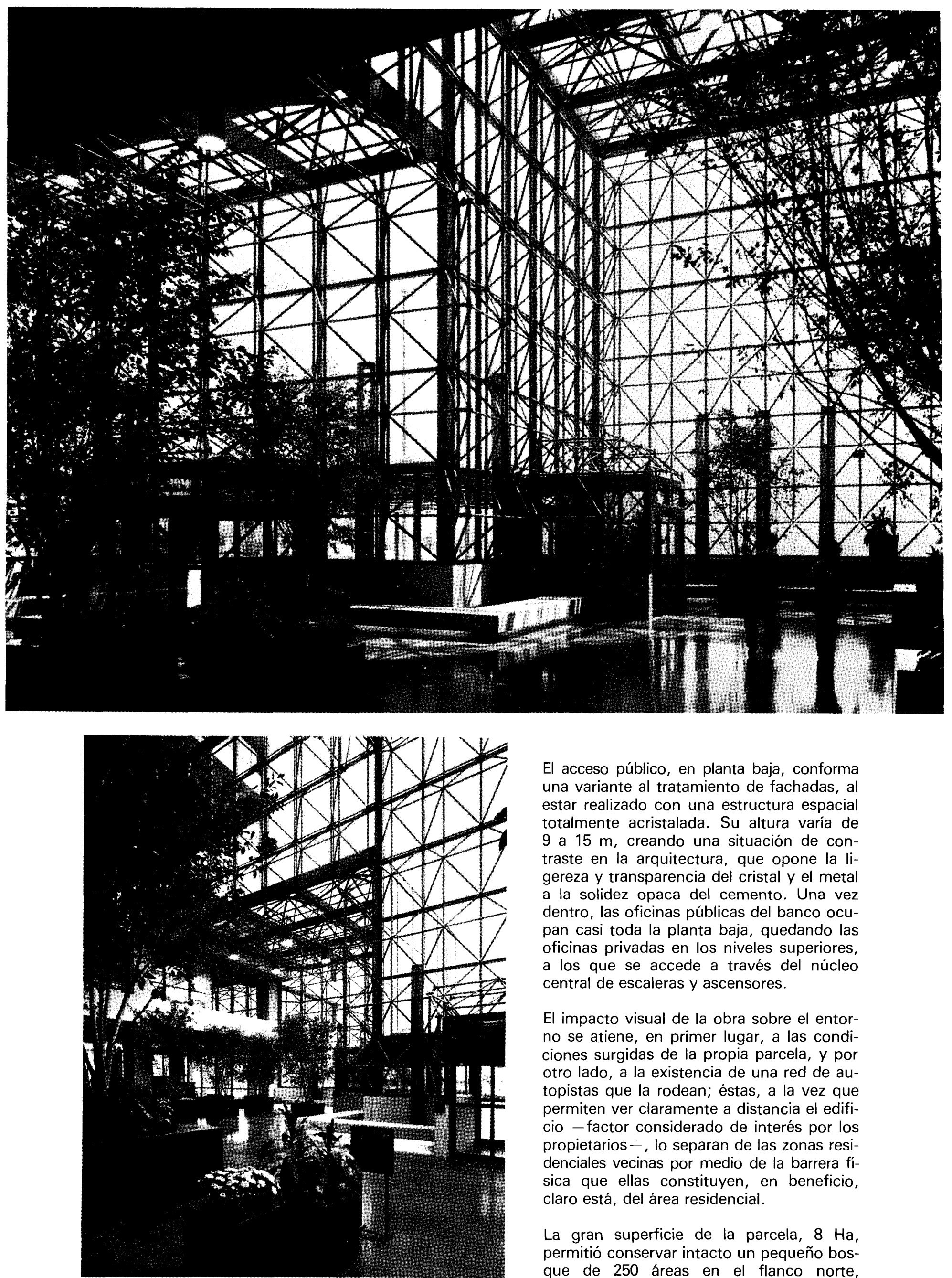

El acceso público, en planta baja, conforma una variante al tratamiento de fachadas, al estar realizado con una estructura espacial totalmente acristalada. Su altura varía de 9 a $15 \mathrm{~m}$, creando una situación de contraste en la arquitectura, que opone la ligereza y transparencia del cristal y el metal a la solidez opaca del cemento. Una vez dentro, las oficinas públicas del banco ocupan casi toda la planta baja, quedando las oficinas privadas en los niveles superiores, a los que se accede a través del núcleo central de escaleras y ascensores.

El impacto visual de la obra sobre el entorno se atiene, en primer lugar, a las condiciones surgidas de la propia parcela, y por otro lado, a la existencia de una red de autopistas que la rodean; éstas, a la vez que permiten ver claramente a distancia el edificio - factor considerado de interés por los propietarios - , lo separan de las zonas residenciales vecinas por medio de la barrera física que ellas constituyen, en beneficio, claro está, del área residencial.

La gran superficie de la parcela, $8 \mathrm{Ha}$, permitió conservar intacto un pequeño bos que de 250 áreas en el flanco norte, 


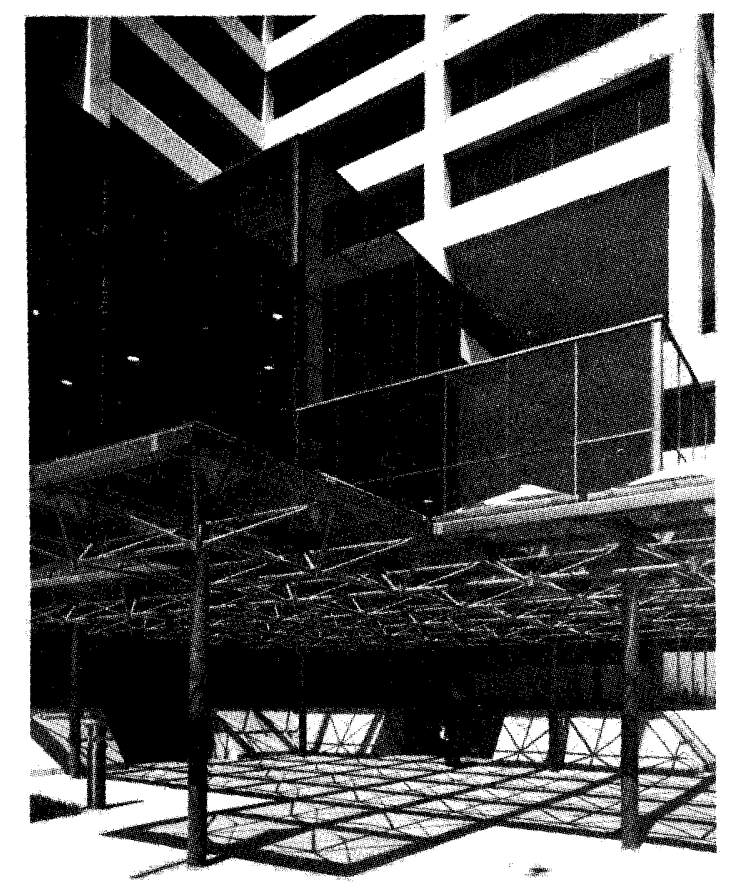

conformando una barrera natural alli donde la parcela lindaba con el área de viviendas. Con la misma finalidad de impedir la distorsión y el obstáculo visual, las 350 plazas de aparcamiento para empleados se sitúan en una explanada a 3,50 m por debajo de la línea de rasante. A este aparcamiento se suma otro, destinado al público, de 912 plazas de capacidad.

El empleo de materiales para el tratamiento de fachadas es sumamente sobrio, comprendiendo paneles prefabricados de cemento, hormigón armado in situ, y metal oscuro en la carpintería exterior. La compartimentación con mamparas de cristal da fluidez y amenidad al espacio interior, permitiendo una abundante luminosidad natural.
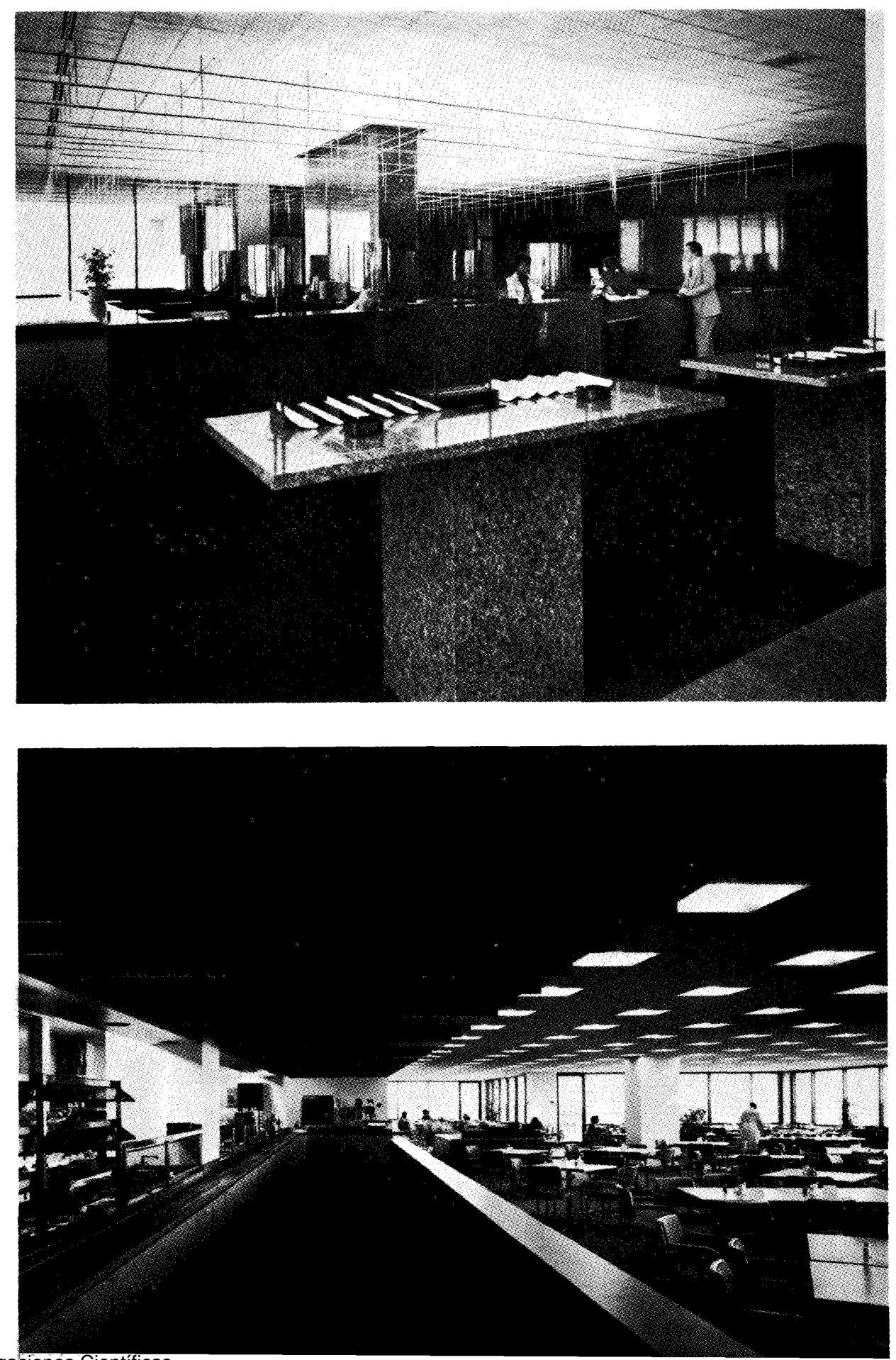
